\title{
Retinoid induction of alveolar regeneration: from mice to man?
}

\author{
M Hind, ${ }^{1,2} \AA$ Gilthorpe, ${ }^{2}$ S Stinchcombe, ${ }^{2}$ M Maden ${ }^{2}$
}

${ }^{1}$ Department of Respiratory Medicine, Royal Brompton Hospital and National Heart and Lung Institute, Imperial College London, London, UK; ${ }^{2} \mathrm{MRC}$ Centre for Developmental Neurobiology, King's College London, London, UK

Correspondence to:

Dr M Hind, Department of Respiratory Medicine, Royal Brompton Hospital and National Heart and Lung Institute, Imperial College London, London SW36NP, UK; m.hind@ imperial.ac.uk

Received 1 August 2008 Accepted 16 October 2008

\section{ABSTRACT}

The use of retinoids to induce human lung regeneration is under investigation in a number of studies in patients with chronic obstructive pulmonary disease (COPD). Retinoic acid (RA) has complex pleiotropic functions during vertebrate patterning and development and can induce regeneration in a number of different organ systems. Studies of retinoid signalling during lung development might provide a molecular basis to explain pharmacological induction of alveolar regeneration in adult models of lung disease. In this review the role of endogenous RA signalling during alveologenesis is explored and data suggesting that a number of exogenous retinoids can induce regeneration in the adult lung are discussed. Current controversies in this area are highlighted and a hypothesis of lung regeneration is put forward. Understanding the cellular and molecular mechanisms of induction of regeneration will be central for effective translation into patients with lung disease and may reveal novel insights into the pathogenesis of alveolar disease and senescence.

The lung is the primary organ for gas exchange across mammalian species, providing an interface between blood and air in a structurally stable environment. As gas exchange is a passive process, a large thin surface area is required for efficient supply of oxygen and removal of carbon dioxide. Gas exchange occurs in alveolar saccules, alveoli and alveolar ducts. Alveolar formation (or alveologenesis) occurs predominantly during a developmental restricted early postnatal period in rats, mice and humans. ${ }^{1-5}$ The cellular and molecular regulation of this complex developmental event is not well understood. Here we discuss a growing body of evidence highlighting the role of endogenous retinoic acid (RA) signalling in the regulation of mammalian alveologenesis, and interpret findings suggesting that pharmacological dosing with RA can induce alveolar regeneration in adult animals outside the temporally restricted period of alveologenesis. As fundamental developmental mechanisms are widely conserved across species, these findings open up the possibility of human lung regeneration as a potential therapeutic approach for patients with lung disease.

The gas exchanging tissues of the pulmonary alveoli are lined with epithelial cells (type I and type II cells) which are in direct contact with air and therefore vulnerable to injury following the inhalation of toxins, infections or reactive oxygen species. The remarkable ability of the lung to maintain function through the life of the organism must, in part, reflect the ability of epithelial, interstitial and endothelial cell populations-together with extracellular matrix - to undergo renewal, repair or regeneration over the lifetime of the animal. In other words, the gas exchanging regions of the lung must have a degree of plasticity. That this plasticity exists has been demonstrated, at least in rodents, by a body of work by Massaro and colleagues and others in a number of different experimental paradigms including re-feeding after calorie restriction $^{6}$ and oestrogen replacement in oestrogendeficient mice, ${ }^{7}$ and is exemplified by experiments showing that exogenous all-trans retinoic acid (atRA) can induce alveolar regeneration in adult rats with elastase-induced emphysema, ${ }^{8}$ partially rescue emphysema in the tight-skin mouse mutant ${ }^{9}$ and rescue dexamethasone (Dex)-impaired alveologenesis in adult mouse lung. ${ }^{10-12}$

The ability to pharmacologically induce alveolar regeneration is a novel and attractive therapeutic approach for patients with too few alveoli and a reduced gas exchanging surface area and might be applied to infants with developmental lung arrest, adults with emphysema or adults with age-related loss of alveoli. ${ }^{13}$ The restoration of functional gas exchanging tissue in humans with alveolar insufficiency would be a significant step forward in the treatment of respiratory diseases that have major public health implications where little in the way of disease-modifying therapy exists. ${ }^{14}$ Whether it is possible to induce regeneration in the human lung is not yet known. Currently, specific retinoic acid receptor (RAR) $\gamma$ agonists are in phase II clinical trials in adults with emphysema secondary to $\alpha_{1}$ antitrypsin deficiency and in patients with smoking-related emphysema.

In this review we discuss the potential mechanisms by which atRA can induce alveolar regeneration in animal models by examining data highlighting the role of endogenous RA signalling that occurs during normal alveolar formation. Deciphering the complex cellular, molecular and genetic signals that regulate development and regeneration provides not only an insight into the pathogenesis of diseases where homeostatic repair processes are disrupted, but should also identify rational targets for the development of future regenerative therapies. In other biological systems, regeneration - at least in part-recapitulates development so, to understand lung regeneration, we must first consider lung development.

\section{LUNG DEVELOPMENT}

Until recently, most studies of mammalian lung development have been performed in rats, ${ }^{2}{ }^{15}$ but the recent explosion in mouse genetics offers a highly manipulated genome which allows specific questions to be asked about the role of candidate developmental genes. These studies, coupled with lineage tracing experiments, should enable progress to be 
made in deciphering the complex patterning mechanisms that underpin lung development. Current knowledge of these mechanisms is, at best, sketchy. Normal function of the lung requires the correct temporal and spatial sequence of developmental mechanisms. Understanding how these fundamental signalling pathways that appear to be active-rather like switching lights on and off at different times in different places-are regulated and interact will be central to understanding not only mechanisms of alveolar development, but also alveolar disease and senescence.

The lung arises from foregut endoderm during early development of the embryo. Paracrine signalling between the extending epithelium and surrounding mesenchyme is involved in patterning the developing lung bud. ${ }^{16}$ Lung development proceeds through four morphologically distinct but continuous embryonic phases that include branching morphogenesis (embryonic day, E9-E12 in mice), the pseudoglandular stage (E12-E15 in mice), canalicular stage (E15-E17 in mice) and saccular stages (E17-birth in mice). In rats and mice, pups are born with no true alveoli but thick-walled alveolar saccules and alveolar formation occurs entirely as a postnatal event. ${ }^{1-3}$ Human alveologenesis occurs from around 24 weeks, the current limit of premature survival, the majority of human alveolar formation occurring during the early postnatal period, certainly up to 18 months $^{5}$ and possibly into later childhood even early adulthood.

\section{A DISTAL LUNG SIGNALLING CENTRE AND INDUCTION OF ALVEOLOGENESIS}

Alveoli are generated through a number of different possible mechanisms that are differentially regulated, the best characterised being alveolar septation where alveolar saccules are subdivided by the eruption of a secondary crest from the primary saccular wall. Septation is a developmentally regulated process that occurs in mice from postnatal day 4 (P4)-P14 (fig 1). The relative timing of alveolar septation is species-dependent and has been proposed to be regulated by oxygen requirements at birth. ${ }^{17}$ Morphological changes driven by proliferation of type II epithelial cells and fibroblasts together with differentiation of type I epithelial cells, transformation of a double capillary system into a single complex capillary network, and apoptosis of specific cell populations resulting in thinning of the alveolar wall and increased architectural complexity characteristic of adult lung tissue.

The molecular signals that induce septal eruption are not yet known, but retinoid signalling in pulmonary lipofibroblasts appears to play a key role. ${ }^{18}$ These cells resemble stellate cells of the liver and are the major retinoid storage cell of the lung. ${ }^{19}$ Lipofibroblasts contain many components of the retinoid signalling pathway including receptors and binding proteins ${ }^{20}$ and can synthesise biologically active RA. ${ }^{21}$ Elastin is a major component of the extracellular matrix with low turnover in steady state and contributes to the elastic properties of the lung. Lipofibroblasts respond in an autocrine manner to RA by upregulation of elastin gene transcription. ${ }^{22}$ Lipofibroblasts are found in septating tissue adjacent to alveolar type II epithelial cells. $^{2}$ In vitro studies demonstrate that RA can induce proliferation in immortalised type II cells in vitro, suggesting a paracrine action of RA on epithelial type II cells during alveologenesis ${ }^{23}$ and a paracrine manner on endothelial cells to induce gene transcription. ${ }^{21}$ Lipofibroblasts are progenitors of myofibroblasts, a subset of fibroblasts that have contractile components that are required for alveolar septation to proceed, ${ }^{24}$ perhaps by facilitating septal elongation. These findings suggest that lipofibroblasts are ideally placed to establish a local morphogenetic signalling centre to coordinate alveolar septal eruption.
Interestingly, morphometric studies suggest that septation alone does not account for the entire increase in alveolar tissue that occurs in the postnatal period, and that alveoli might be generated by the transformation of distal bronchioles into alveoli, called retrograde alveolisation. ${ }^{25}$ Recently, a subpopulation of cells known as bronchoalveolar stem cells (BASCs) has been identified that are located at the interface between the terminal bronchiole and the alveolar epithelium - the bronchioalveolar duct junction. These cells co-express Clara cell markers (CC10) typical of distal bronchiolar epithelium and type II cell markers (SP-C) seen in the alveolar epithelium. Isolated BASC populations in vitro have been shown to self-renew - a requirement for a stem cell populationand can differentiate into cells that express markers characteristic of bronchial epithelium, alveolar type I cells or alveolar type II cells. ${ }^{26}$ The role of these cells in alveolar formation has not yet been defined, but the location and multilineage potential of this population suggests that, under the right developmental cues, these cells might contribute to alveologenesis.

There is some evidence that matrix turnover is higher in subpleural regions of the lung outside the period of septation, and this is accompanied by a shift in the location of lipofibroblasts from central regions during septation to the periphery after septation ceases. ${ }^{27}$ In studies of alveolar wall cell proliferation during murine alveologenesis, two discrete patterns of proliferating cells have been identified: a central pattern that is temporally associated with septation and a peripheral subpleural pattern that extends into adulthood. ${ }^{28}$

\section{ENDOGENOUS RETINOIDS AND RA SYNTHESIS IN THE POSTNATAL MOUSE LUNG}

Retinoids are the biologically active metabolites of vitamin A (retinol) and have a central role in a number of developing and regenerating biological systems including developing and regenerating limb, skin and central nervous system. ${ }^{29}$ In these quite different models, common themes emerge to explain exogenous effects of retinoids by the endogenous effects of RA during development. ${ }^{30}$

Mammals are unable to synthesise retinoids de novo and are dependent on dietary sources either in the form of $\beta$-carotenes from plants or retinyl esters from animal sources. Following absorption from the gut, retinoids are stored in a number of organs prior to utilisation. The lung is second only to the liver as the major retinoid storage organ. The requirement of retinoids for the integrity of epithelial tissues, including the lung, has been known about for many years. ${ }^{31}$ Adult lung contains a wide range of different retinoids including all-trans RA (atRA), 13-cis RA, 14,4di-dehydroretro RA, 4-oxoRA, anhydroretinol and retinyl esters. ${ }^{28}$ The availability of active RA is modulated by a number of factors including the distribution and activity of RA synthesising enzymes and RA modulating enzymes including cytochrome P450 enzymes such as CYP26 1a1 and CYP26 1b1. The retinaldehyde dehydrogenase enzymes (RALDHs) are the rate-limiting step in local RA production, and their spatial distribution in the embryo usually defines RA-dependent regions or periods of development. ${ }^{29}$

In addition to endogenous retinoids, lung tissue contains RALDH enzymes that are temporally and spatially regulated during alveologenesis in the mouse (RALDH-1 and RALDH-2). This study suggests the expression of different RALDH enzymes are associated with different patterns of alveolar wall cell proliferation; RALDH-1 is associated with the tightly regulated central pattern corresponding to the period of alveolar septation and RALDH-2 with a peripheral subpleural pattern supportive of the circumferential addition of alveoli. RALDH-1 is located in bronchial epithelial cells and in cells within the alveolar wall but 

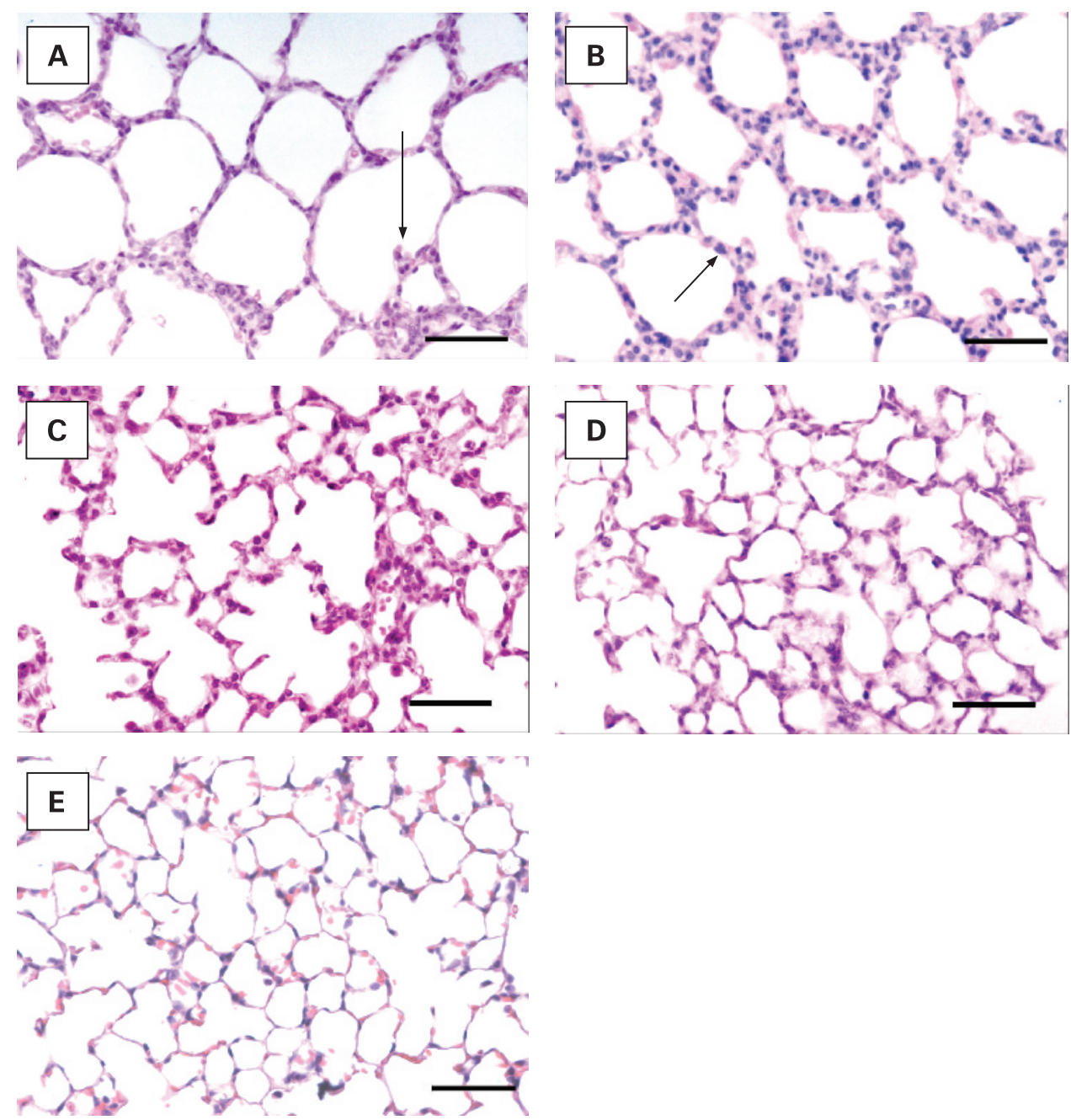

Figure 1 Increasing alveolar complexity during postnatal alveolar formation in the mouse lung. High power images of the developing postnatal mouse lung. All images are taken at the same magnification. (A) The smooth walls of the large alveolar saccules are clearly visible in the P1 mouse lung. (B) With subsequent alveolar development to P4, the saccules become subdivided by the eruption of thick-walled primary alveolar septa (arrows). (C) At P9, numerous secondary septa erupt from the primary septal wall (arrowheads). (D) At P15, the alveoli have thinner alveolar walls and resemble the structure of the adult lung (E). Scale bar $50 \mu \mathrm{m}$. P, postnatal day.

not pleural tissue. RALDH-2 is identified in bronchial epithelial tissue and pleura but not in the alveolar septum. ${ }^{28}$

\section{RETINOID BINDING PROTEINS DURING MURINE ALVEOLOGENESIS}

Both retinol and RA are bound in the cytoplasm by binding proteins - cellular retinol binding protein (CRBP-I and -II) and cellular retinoic acid binding protein (CRABP-I and -II). These families of cytoplasmic binding proteins regulate the biological action of retinol and RA, modulating both the availability of free retinoid and ensuring specificity between the retinoid and its metabolic enzymes (fig 2). ${ }^{33}$ Previous Northern blot analysis of CRBP-I and CRABP-I mRNA has demonstrated upregulation of both genes during alveologenesis in both whole rat lung tissue and isolated lipid-laden fibroblasts. ${ }^{20}$ Dexamethasone administered to postnatal rat pups between P4 and P14 disrupts alveolar septation ${ }^{34}$ and results in downregulation of CRBP-I and CRABPII. ${ }^{35}$ Retinoid binding proteins are located in parenchymal lung tissue during alveologenesis. ${ }^{36}$ The temporal rise in the CRBP-I and CRABP-I towards the end of alveolar septation in both rats and mice suggests that these proteins might regulate the availability of free retinol and RA to end septation.

\section{RETINOIC ACID RECEPTORS DURING MURINE ALVEOLOGENESIS}

RA exerts its effects via nuclear receptors, retinoic acid receptors (RARs) which act as ligand-activated transcription factors to alter the pattern of gene transcription of a cell. There are three subtypes of $\operatorname{RAR}(\alpha, \beta$ and $\gamma$ ), each of which has a number of isoforms through differential promoter usage and splice variants. There are seven isoforms of $\operatorname{RAR} \alpha$, four isoforms of $\operatorname{RAR} \beta$ and seven isoforms of RAR $\gamma$. In the mouse, RAR $\alpha 1, \operatorname{RAR} \beta 2, \operatorname{RAR} \beta 4$ and $\operatorname{RAR} \gamma$ have been identified in postnatal lung tissue and expression of these receptors changes over time. ${ }^{36}$ These data are in agreement with earlier experiments identifying $\operatorname{RAR} \alpha, \beta$ and $\gamma$ in lipofibroblasts isolated from rat lung. ${ }^{20}$

The function of the individual RARs on alveolar formation has been investigated in mice using knockouts of each of the three RARs. Morphometric study of RAR $\alpha-/-$ mice found no difference between control and knockout animals at P14 (the end of alveolar septation), but at P50 both the number of alveoli and surface area is reduced compared with controls which suggests that RAR $\alpha$ has no effect on septation but has a role on non-septation alveologenesis. ${ }^{37}$ The effect of RAR $\beta$ on alveolar formation has been determined by analysis of gene deletions and by use of specific RAR $\beta$ agonists ${ }^{38}$ which show that 

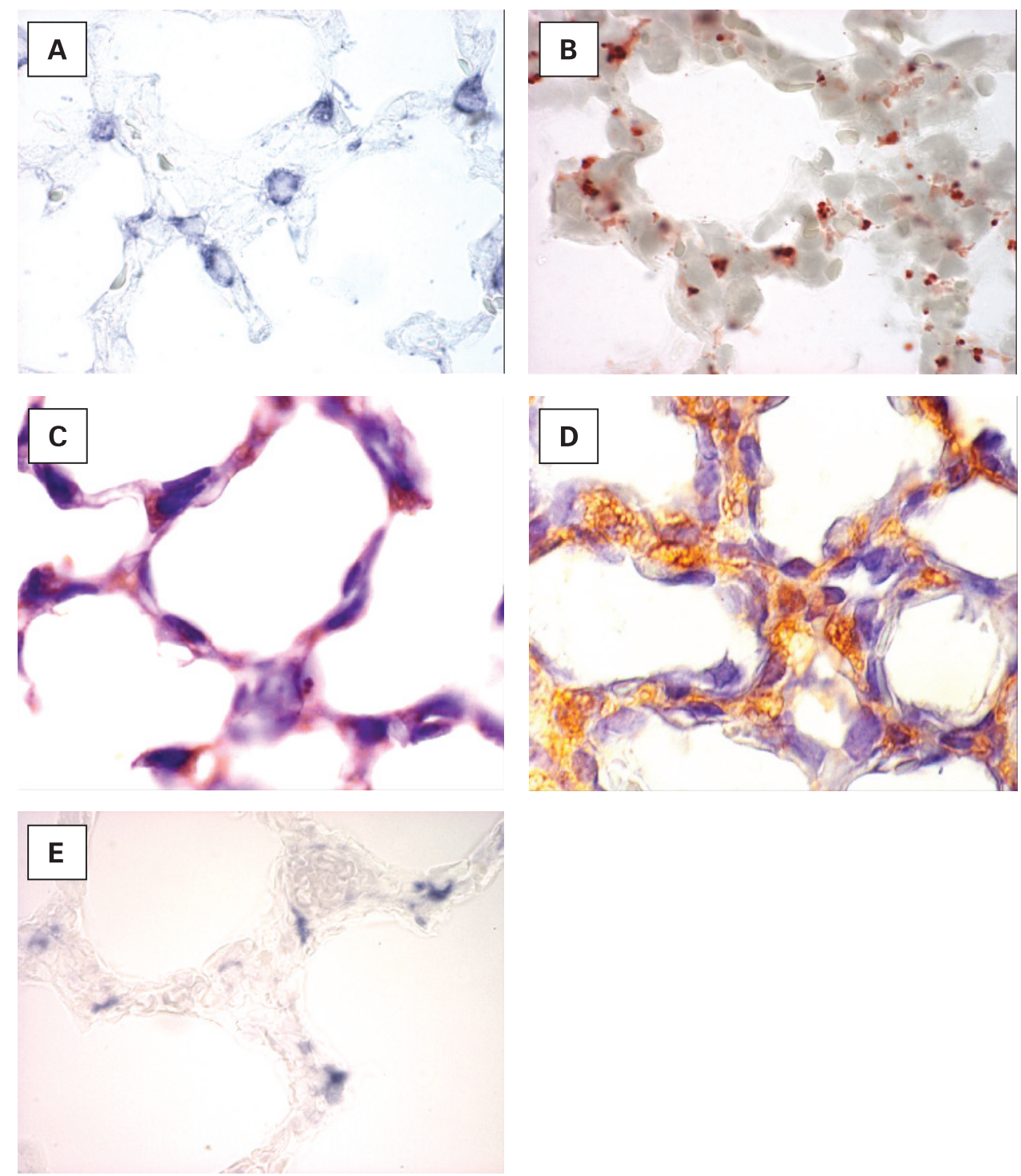

Figure 2 Type II cells, lipofibroblasts, retinoic acid (RA) signalling proteins and elastin mRNA expression during murine alveologenesis. Cell types and RA signalling proteins associated with alveologenesis. (A) In situ hybridisation with digoxigenin-labelled antisense riboprobes to surfactant protein $C$ mRNA (blue colour) restricted to type II pneumocytes. These cells proliferate in response to RA in vitro. (B) Oil red-0 highlights the presence of lipid droplets (red stain) in retinoid-containing lipofibroblasts on frozen sections of alveolar parenchyma. (C) Immunohistochemistry demonstrating retinaldehyde dehydrogenase-1 (RALDH-1) protein (brown staining), an endogenous RA-synthesising enzyme in specific alveolar parenchymal cells. (D) Cellular retinol binding protein (CRBP-1) immunohistochemistry (brown stain) reveals widespread alveolar parenchymal distribution of the retinoid binding proteins. (E) In situ hybridisation with digoxigenin-labelled antisense riboprobes (blue colour) to tropoelastin demonstrating asymmetrical tropoelastin mRNA expression, an RA regulated target gene, in the presumptive erupting alveolar septa.

RAR $\beta-/$ - animals septate earlier and at a faster rate than controls, and that dosing with RAR $\beta$-specific agonists impairs septation. These data suggest that $\operatorname{RAR} \beta$ is a negative regulator of septation although, interestingly, analysis of a different RAR $\beta$ mutant gave different results with a reduction in the gas exchanging surface area per lung volume. ${ }^{39}$ Deletion of one RAR $\gamma$ allele has been found in the compound RXR/RAR $\gamma+/-$ mice to result in a reduction in whole lung elastic tissue, number of alveoli and an increase in the distance between alveolar walls and mean chord length (Lm). In addition, elastin transcription in lipofibroblasts was reduced at $\mathrm{P} 14 .{ }^{40}$ These data suggest that RAR $\gamma$ is a positive regulator of septation.

\section{RETINOID SIGNALLING IN HUMAN ALVEOLOGENESIS}

From the data above, numerous lines of evidence point to a central role of RA signalling in alveolar formation in rodents. Is there any evidence that this developmental mechanism is conserved in man? Chronic lung disease of prematurity or bronchopulmonary dysplasia (BPD) describes a clinical syndrome of lung dysfunction characterised by a developmental lung arrest. ${ }^{41}$ The aetiology of $\mathrm{BPD}$ is multifactorial with prematurity, infection, mechanical ventilation, oxygen toxicity and the use of glucocorticoids all implicated. Low serum vitamin A (retinol) levels have been associated with BPD. ${ }^{42}$ Intermittent three times weekly dosing with retinol is one of the few interventions found to have a small but significant effect on reducing the incidence of BPD in extremely low birthweight infants, ${ }^{43}$ although the mechanism of this effect is not known. Interestingly, the first human mutation of RA signalling (STRA6), the recently discovered membrane receptor for uptake of circulating retinol, ${ }^{44}$ has been described in two families following a screen of infants with anophthalmos. These infants had a number of specific developmental malforma- 
tions including lung hypoplasia, suggesting a requirement for correct RA signalling in human alveolar development. ${ }^{45}$

These findings suggest an evolutionary conserved pathway in which developmentally regulated endogenous RA is synthesised from stored retinoids in lipofibroblasts to establish a distal lung signalling centre. This epithelial-mesenchymal signalling centre acts in an autocrine manner on lipofibroblasts with paracrine actions on type II cells, endothelial cells and myofibroblasts to initiate-and perhaps coordinate-alveolar septal eruption and regulate alveologenesis. This model provides a molecular framework to consider the effects of exogenous RA on the induction of regeneration.

To determine whether the Dex effect also occurs in the mouse, neonatal mouse pups were dosed from P2-P14 with Dex or dilutent and allowed to develop into adults with no further intervention. This generates adult mice in which alveolar development has been disrupted and spontaneous alveolar regeneration does not occur (at least until P150). ${ }^{52}$ To investigate the physiology of this model we used spontaneously breathing, whole body plethysmography under conditions of physiological stress to demonstrate that neonatal Dex dosing results in increased tidal volume, minute volume and a reduced rate in adult animals (ie, Dex-dosed animals breathe more slowly, for longer and work harder; Stinchcombe et al, unpublished). The structural and functional characteristics of this model of disrupted development share many characteristics with human BPD/COPD.

Using this Dex model it has been shown that 10 doses of RA from P40-P52 results in changes in morphology and morphometry suggestive of successful alveolar regeneration (a reduction in Lm and increase in surface area to that of control animals) when assayed at $\mathrm{P} 90 .{ }^{10}$ Using this model of airspace enlargement, other molecules can be assayed for potential regenerative effects. We have tested eight other retinoids including 13-cis RA, 9-cis RA, retinol, 4-oxo RA, a pan RXR agonist and $R A R \alpha$, RAR $\beta$ and RAR $\gamma$ agonists in our murine Dex model, some of which induce regeneration ( $\operatorname{RAR} \alpha, \operatorname{RAR} \gamma, 4$-oxo $\mathrm{RA}$ ) but others do not (13-cis RA, retinol, pan-RXR agonist) (fig 3). ${ }^{11}$ Using the Dex/RA protocol and manipulating the genetic background of the mouse, we can determine which downstream genes are required for regeneration to occur and which are redundant. For example, RA can induce regeneration following the Dex/RA

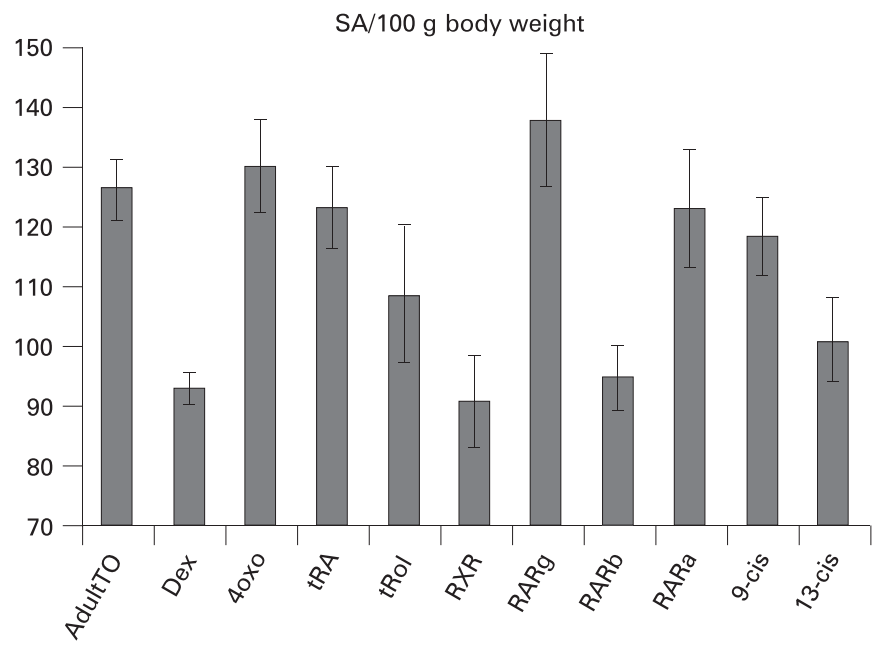

Figure 3 Induction of regeneration using different retinoids. Restoration of gas exchanging surface area (SA) normalised to body weight in the dexamethasone (Dex) mouse model of alveolar insufficiency: 4-oxo RA, atRA, RAR $\gamma$ agonist, RAR $\alpha$ agonist and 9-cisRA restore lost SA but retinol, pan-RXR agonists, RAR $\beta$ agonists and 13-cis RA do not. protocol on an RAR $\beta$ null mutant background, suggesting that $\operatorname{RAR} \beta$ is not required for the regenerative effect. This finding is confirmed by data demonstrating that RAR $\beta$-specific agonists do not induce regeneration in wild-type mice. ${ }^{11}$ This combination of morphometry, genetics and experimental intervention provides a potential approach for analysis of microarrayidentified downstream regeneration genes.

\section{RA-INDUCED ALVEOLAR REGENERATION: CURRENT CONTROVERSIES}

Given the widespread potential implications of lung regeneration, numerous studies have tried to replicate the original report by Massaro and Massaro ${ }^{8}$ in different animal models of disease. To date, seven have succeeded ${ }^{10} 11^{385-55}$ and in two other experimental paradigms - pneumonectomy and oxygen damage - atRA stimulated proliferation or protected from damage. ${ }^{56}{ }^{57}$ However, a similar number of studies have failed to show any beneficial effects of atRA. ${ }^{58-64}$ The reason for these differences are not yet clear.

To investigate why some of the published studies using mice failed to identify a regenerative effect, we investigated the effect of background strain on regeneration. Our original studies were performed in the outbred TO strain but, in these experiments, we examined inbred NIHS and ICR strains. ${ }^{65}$ Using the same protocol as described above, NIHS mice gave similar results to the TOs (ie, a mean decrease in Lm in Dex-treated animals to atRA-treated animals which represents a $56 \%$ recovery towards the control Lm value). The same distribution of animals in the atRA-treated group was observed with some showing complete recovery, the majority showing a partial response and some showing no response at all. However, using this protocol, the ICR strain revealed that atRA had absolutely no effect on alveolar structure or morphometry. When the above experiment was repeated on the ICR strain but with a five times higher administered dose of atRA (10 mg/kg), successful regeneration was induced. These data suggest that $\mathrm{RA}$-induced regeneration is not a strain-specific phenomenon but that there are important differences in RA pharmacokinetics between strains.

The second obvious clinical scenario where RA might have therapeutic implications is in adults with emphysema. Epidemiological data suggest that there is an inverse relationship between serum vitamin A levels and lung function in smokers with COPD. ${ }^{68}$ The NIH-funded FORTE study (Feasibility of Retinoids for the Treatment of Emphysema) investigated dosing with atRA $1 \mathrm{mg} / \mathrm{kg}, 2 \mathrm{mg} / \mathrm{kg}$ or 13-cis RA in a 6-month trial with a 3-month crossover period and subsequent 18-month observation period. ${ }^{69}$ Retinoids were generally well tolerated in this patient group with only minor side effects (commonly rash, hypertriglyceridaemia and mild alterations in liver function tests). There were no significant improvements in the CT densitometry score, gas transfer or quality of life score at the end of this feasibility study, but there was evidence of some biological activity, with a change in carbon monoxide transfer factor (initial decline with later recovery) in patients who received the highest doses of RA with high plasma levels. The significance of this finding is as yet unclear. atRA can induce pulmonary toxicity as an acute retinoic acid syndrome. Unfortunately, the crossover design of the FORTE trial resulted in no true placebo group for long-term follow-up. The reported change in carbon monoxide transfer factor could therefore represent either drug-induced toxicity or an alveolar remodelling process.

Specific RAR $\gamma$ selective agonists are currently in longer Pharma-sponsored placebo controlled phase II clinical trials in patients with emphysema secondary to $\alpha_{1}$-antitrypsin deficiency and in patients with moderately severe COPD due to smoking. These molecules are at least as potent as atRA at inducing 
alveolar regeneration in the Dex mouse model, do not induce their own metabolism and have more predictable pharmacokinetics so that higher drug levels can be maintained. The outcome of these trials is expected in the next few years. These studies highlight the usefulness of appropriate animal models in the rational design of candidate regenerative therapies for the treatment of human lung disease.

The induction of endogenous repair or regenerative pathways using exogenous agents represents a promising and exciting therapeutic approach for patients with lung disease. The development of a mouse model allows the cellular and molecular biology of the induction of alveolar regeneration to be studied in detail. Translational studies with the goal of inducing lung regeneration are now underway in patients with emphysema. Just 10-15 years ago the idea that the adult lung had the capacity to undergo regeneration was viewed with scepticism. The fact that human trials are now underway is remarkable. The next 10 15 years' research promises huge advances in this field.

Funding: This work was supported by a Wellcome Training Fellowship (MH), a Wellcome Trust project grant (MM and MH), Wellcome VIP Award (AG) and an MRC Clinical Fellowship (SS).

Competing interests: None.

\section{REFERENCES}

1. Amy RW, Bowes D, Burri PH, et al. Postnatal growth of the mouse lung. J Anat 1977:124(Pt 1):131-51

2. Burri PH. The postnatal growth of the rat lung. 3. Morphology. Anat Rec 1974; 180:77-98

3. Burri PH, Dbaly J, Weibel ER. The postnatal growth of the rat lung. I. Morphometry. Anat Rec 1974;178:711-30.

4. Kauffman SL, Burri PH, Weibel ER. The postnatal growth of the rat lung. II. Autoradiography. Anat Rec 1974:180:63-76.

5. Zeltner TB, Burri PH. The postnatal development and growth of the human lung. II. Morphology. Respir Physiol 1987;67:269-82

6. Massaro D, Massaro GD. Hunger disease and pulmonary alveoli. Am J Respir Crit Care Med 2004;170:723-4.

7. Massaro D, Massaro GD. Estrogen regulates pulmonary alveolar formation, loss, and regeneration in mice. Am J Physiol Lung Cell Mol Physiol 2004;287:L1154-9.

8. Massaro GD, Massaro D. Retinoic acid treatment abrogates elastase-induced pulmonary emphysema in rats. Nat Med 1997:3:675-7.

9. Massaro G, Massaro D. Retinoic acid partially rescues failed septation in rats and in mice. Am J Physiol Lung Cell Mol Physiol 2000;278:L955-60.

10. Hind $\mathbf{M}$, Maden M. Retinoic acid induces alveolar regeneration in the adult mouse lung. Eur Respir J 2004;23:20-7

11. Maden M. Retinoids have differing efficacies on alveolar regeneration in a dexamethasone-treated mouse. Am J Respir Cell Mol Biol 2006;35:260-7.

12. Stinchcombe SV, Maden M. Retinoic acid induced alveolar regeneration: critical differences in strain sensitivity. Am J Respir Cell Mol Biol 2008;38:185-91.

13. Massaro D, Massaro GD. Toward therapeutic pulmonary alveolar regeneration in humans. Proc Am Thorac Soc 2006;3:709-12.

14. Murray CJL, Lopez AD. The global burden of disease. A comprehensive assesment of mortality and disablilty from diseases, injuries and risk factors in 1990 and projected to 2020. Cambridge, Massachusetts: World Health Organization, 1996.

15. Massaro D, Teich N, Maxwell S, et al. Postnatal development of pulmonary alveoli: regulation and evidence for a "critical period" in rats. J Clin Invest 1985;76:1297-305.

16. Warburton D, Schwarz M, Tefft D, et al. The molecular basis of lung morphogenesis Mech Dev 2000;92:26.

17. Massaro D, Massaro GD. Invited review: pulmonary alveoli: formation, the "call for oxygen," and other regulators. Am J Physiol Lung Cell Mol Physiol 2002;282:L345-58.

18. McGowan SE, Torday JS. The pulmonary lipofibroblast (lipid interstitial cell) and its contributions to alveolar development. Annu Rev Physiol 1997:59:43-62.

19. Okabe T, Yorifuji H, Yamada E, et al. Isolation and characterization of vitamin-Astoring lung cells. Exp Cell Res 1984;154:10.

20. McGowan SE, Harvey CS, Jackson SK. Retinoids, retinoic acid receptors, and cytoplasmic retinoid binding proteins in perinatal rat lung fibroblasts. Am J Physiol 1995;269(4 Pt 1):L463-72.

21. Dirami G, Massaro GD, Clerch LB, et al. Lung retinol storing cells synthesize and secrete retinoic acid, an inducer of alveolus formation. Am J Physiol Lung Cell Mol Physiol 2004;286:L249-56.

22. McGowan SE, Doro MM, Jackson SK. Endogenous retinoids increase perinatal elastin gene expression in rat lung fibroblasts and fetal explants. Am J Physiol 1997;273(2 Pt 1):L410-6.

23. Nabeyrat E, Corroyer S, Epaud R, et al. Retinoic acid induced proliferation of lung alveolar epithelial cells: relation with the IGF system. Am J Physiol Lung Cell Mol Physiol 1998;275:L71-9.
24. Lindahl P, Karlsson L, Hellstrom M, et al. Alveogenesis failure in PDGF-A-deficient mice is coupled to lack of distal spreading of alveolar smooth muscle cell progenitors during lung development. Development 1997;124:3943-53.

25. Randell S, Mercer R, Young S. Postnatal growth of pulmonary acini and alveoli in normal and oxygen-exposed rats studied by serial section reconstruction. Am J Anat 1989;186:55-68.

26. Kim C, Jackson EL, Woolfenden AE, et al. Identification of bronchioalveolar stem cells in normal lung and in lung cancer. Cell 2005;121:823-35.

27. Massaro GD, Massaro D. Postnatal lung growth: evidence that the gas-exchange region grows fastest at the periphery. Am J Physiol 1993;265(4 Pt 1):L319-22.

28. Hind M, Corcoran J, Maden M. Alveolar proliferation, retinoid synthesizing enzymes, and endogenous retinoids in the postnatal mouse lung. Different roles for Aldh-1 and Raldh-2. Am J Respir Cell Mol Biol 2002;26:67-73.

29. Maden M. Retinoid signalling in the development of the central nervous system. Nat Rev Neurosci 2002:3:843-53.

30. Maden M, Hind M. Retinoic acid, a regeneration-inducing molecule. Dev Dyn 2003:226:237-44.

31. Wolbach SB, Howe PR. Tissue changes following deprivation of fat-soluble A vitamin. J Exp Med 1925;42:753-77.

32. Wolbach SB, Howe PR. Epithelial repair and recovery from vitamin A deficiency. $J$ Exp Med 1933;57:511-26.

33. Napoli JL. Interactions of retinoid binding proteins and enzymes in retinoid metabolism. Biochem Biophys Acta 1999;1440:139-62.

34. Massaro D, Teich N, Maxwell S, et al. Postnatal development of alveoli. Regulation and evidence for a critical period in rats. J Clin Invest 1985;76:1297-305.

35. Whitney D, Massaro GD, Massaro D, et al. Gene expression of cellular retinoidbinding proteins: modulation by retinoic acid and dexamethasone in postnatal rat lung. Pediatr Res 1999:45:2-7.

36. Hind M, Corcoran J, Maden M. Temporal/spatial expression of retinoid binding proteins and RAR isoforms in the postnatal lung. Am J Physiol Lung Cell Mol Physiol 2002:282:L468-76.

37. Massaro GD, Massaro D, Chambon P. Retinoic acid receptor-alpha regulates pulmonary alveolus formation in mice after, but not during, perinatal period. Am J Physiol Lung Cell Mol Physiol 2003;284:L431-3.

38. Massaro GD, Massaro D, Chan WY, et al. Retinoic acid receptor-beta: an endogenous inhibitor of the perinatal formation of pulmonary alveoli. Physiol Genomics 2000;4:51-7.

39. Snyder JM, Jenkins-Moore M, Jackson SK, et al. Alveolarization in retinoic acid receptor-beta-deficient mice. Pediatr Res 2005;57:384-91.

40. McGowan S, Jackson SK, Jenkins-Moore M, et al. Mice bearing deletions of retinoic acid receptors demonstrate reduced lung elastin and alveolar numbers. Am J Respir Cell Mol Biol 2000;23:162-7.

41. Jobe AH, Bancalari E. Bronchopulmonary dysplasia: NICHD/NHLBI/ORD workshop summary. Am J Respir Crit Care Med 2001;163:1723-9.

42. Shenai JP, Chytil F, Stahlman MT. Vitamin A status of neonates with bronchopulmonary dysplasia. Pediatr Res 1985;19:185-8.

43. Tyson JE, Wright LL, Oh W, et al. Vitamin A supplementation for extremely-lowbirth-weight infants. National Institute of Child Health and Human Development Neonatal Research Network. N Engl J Med 1999;340:1962-8.

44. Kawaguchi R, Yu J, Honda J, et al. A membrane receptor for retinol binding protein mediates cellular uptake of vitamin A. Science 2007;315:820-5

45. Pasutto F, Sticht H, Hammersen G, et al. Mutations in STRA6 cause a broad spectrum of malformations including anophthalmia, congenital heart defects, diaphragmatic hernia, alveolar capillary dysplasia, lung hypoplasia, and mental retardation. Am J Hum Genet 2007;80:550-60.

46. Weismann A. Versuche uber regeneration bei Tritonen. Anatomischer Anzeiger 1903;22:425-31.

47. Solopaev B. On the problem of regeneration of the lungs in caudate amphibia. USSR Academy of Medical Sciences, 1957

48. Henning SJ. Plasma concentrations of total and free corticosterone during development in the rat. Am J Physiol Endocrinol Metab 1978;238:E415-56.

49. Henning SJ. Postnatal development: coordination of feeding, digestion and metabolism. Am J Physiol 1981;241:G199-214.

50. Massaro D, Massaro GD. Dexamethasone accelerates postnatal alveolar wall thinning and alters wall composition. Am J Physiol 1986;251(2 Pt 2):R218-24.

51. Rush MG, UI-Haq R, Chytil F. Opposing effects of retinoic acid and dexamethasone on cellular retinol-binding protein ribonucleic acid levels in the rat. Endocrinology 1991; 129:705-9.

52. Maden M, Hind M. Retinoic acid in alveolar development, maintenance and regeneration. Phil Trans R Soc Lond B Biol Sci 2004;359:799-808.

53. Belloni PN, Garvin L, Mao CP, et al. Effects of all-trans-retinoic acid in promoting alveolar repair. Chest 2000;117:6.

54. Ishizawa K, Kubo H, Yamada $\mathrm{M}$, et al. Bone marrow derived cells contribute to lung regeneration after elastase-induced pulmonary emphysema. FEBS Lett 2004:556:249-52.

55. Tepper J, Pfeiffer J, Aldrich M, et al. Can retinoic acid ameliorate the physiologic and morphologic effects of elastase instillation in the rat? Chest 2000;117(Suppl):2.

56. Kaza AK, Kron IL, Kern JA, et al. Retinoic acid enhances lung growth after pneumonectomy. Ann Thorac Surg 2001;71:1645-50.

57. Veness-Meehan KA, Pierce RA, Moats-Staats BM, et al. Retinoic acid attenuates $\mathrm{O}_{2}$ induced inhibition of lung septation. Am J Physiol Lung Cell Mol Physiol 2002;283:L971-80.

58. Fujita $\mathbf{M}, Y e \mathrm{Q}$, Ouchi $\mathrm{H}$, et al. Retinoic acid fails to reverse emphysema in adult mouse models. Thorax 2004;59:224-30. 
59. Lucey EC, Goldstein RH, Breuer R, et al. Retinoic acid does not affect alveolar septation in adult FVB mice with elastase-induced emphysema. Respiration 2003;70:200-5.

60. March TH, Bowen LE, Finch GL, et al. Effects of strain and treatment with inhaled all-trans-retinoic acid on cigarette smoke-induced pulmonary emphysema in mice. COPD 2005;2:289-302

61. March TH, Cossey PY, Esparza DC, et al. Inhalation administration of all-transretinoic acid for treatment of elastase-induced pulmonary emphysema in Fischer 344 rats. Exp Lung Res 2004;30:383-404.

62. Meshi B, Vitalis TZ, lonescu D, et al. Emphysematous lung destruction by cigarette smoke. The effects of latent adenoviral infection on the lung inflammatory response. Am J Respir Cell Mol Biol 2002;26:52-7.

63. Nishi Y, Boswell V, Ansari $T$, et al. Elastase-induced changes in lung function: relationship to morphometry and effect of drugs. Pulm Pharmacol Ther 2003;16:221-9.
64. Srinivasan G, Bruce EN, Houtz PK, et al. Dexamethasone-induced changes in lung function are not prevented by concomitant treatment with retinoic acid. Am J Physio Lung Cell Mol Physiol 2002;283:L275-87.

65. Stinchcombe SV, Maden M. Retinoic acid-induced alveolar regeneration: critical differences in strain sensitivity. Am J Respir Cell Mol Biol 2008;38:185-91.

66. Yang L, Naltner A, Yan C. Overexpression of dominant negative retinoic acid receptor alpha causes alveolar abnormality in transgenic neonatal lungs. Endocrinology 2003; 144:3004-11

67. Shenai JP, Kennedy KA, Chytil F, et al. Clinical trial of vitamin A supplementation in infants susceptible to bronchopulmonary dysplasia. J Pediatr 1987;111:269-77.

68. Morabia A, Menkes MJ, Comstock GW, et al. Serum retinol and airway obstruction. Am J Epidemiol 1990;132:77-82.

69. Roth MD, Connett JE, D'Armiento JM, et al. Feasibility of retinoids for the treatment of emphysema study. Chest 2006;130:1334-45.

\section{Pulmonary puzzle}

\section{ANSWERS}

From the questions on page 398

The combination of cystic lung disease, a family history of lung disease and the characteristic papular lesions on this patient are highly suggestive of Birt-Hogg-Dube syndrome (BHDS). Genetic sequencing of the folliculin (FLCN) gene identified a novel frameshift mutation and confirmed the presence of BHDS.

Cystic lung disease occurs in a relatively narrow disease spectrum including lymphangioleimyomatosis/tuberous sclerosis complex, Langerhans cell histiocytosis, lymphocytic interstitial pneumonia, neurofibromatosis, Marfan's syndrome, Ehlers-Danlos syndrome (EDS), $\alpha_{1}$ antitrypsin deficiency, Pneumocystis jirovecii infection and BHDS. This patient's strong family history across several generations suggested an autosomal-dominant process such as neurofibromatosis, Marfan's syndrome, EDS or BHDS. While he did not have the phenotypic characteristics of neurofibromatosis, Marfan's syndrome or EDS, his skin lesions appeared typical for BHDS. Diagnostic evaluation therefore proceeded to confirm BHDS.

BHDS was initially described in 1977 as an autosomal-dominant disorder of benign skin tumours including fibrofolliculomas, trichodiscomas and acrochordons. ${ }^{1}$ Subsequent studies have shown that mutations in the FLCN gene are responsible for BHDS and the gene is highly expressed in skin, kidney and lung stromal cells and type I pneumocytes. ${ }^{2}$ A genotype-phenotype association has been reported between the degree of cystic lung disease, the risk of spontaneous pneumothorax and the exonic location of the FLCN mutation. ${ }^{3}$

Although pneumothoraces in BHDS can be associated with morbidity, mortality may be increased in BHDS due to the development of renal tumours (oncocytic hybrid tumours, chromophobe renal cell carcinoma, clear cell carcinoma, papillary renal cell carcinoma). ${ }^{4}$ In a cross-sectional analysis of 98 patients with BHDS, the age-adjusted odds ratio for the development of renal cell carcinoma was 6.9, with chromophobe renal cell carcinoma being the most common cell type. ${ }^{5}$

Thus, BHDS consists of characteristic benign skin lesions, cystic lung disease with spontaneous pneumothorax and a risk of renal cell carcinoma. Owing to the potential mortality associated with renal tumours, an annual abdominal CT scan is recommended to evaluate the development of these tumours. Furthermore, due to incomplete skin lesion penetrance, family members should be screened with genetic testing or renal ultrasound to check for renal tumours and genetic counselling should be provided to couples planning to have children.

Thorax 2009;64:457. doi:10.1136/thx.2008.102095a

\section{REFERENCES}

1. Birt AR, Hogg GR, Dube WJ. Hereditary multiple fibrofolliculomas with trichodiscomas and acrochordons. Arch Dermatol 1977:113:1674-7.

2. Nickerson ML, Warren MB, Toro JR, et al. Mutations in a novel gene lead to kidney tumors, lung wall defects, and benign tumors of the hair follicle in patients with the Birt-Hogg-Dube syndrome. Cancer Cell 2002;2:157-64.

3. Toro JR, Pautler SE, Stewart L, et al. Lung cysts, spontaneous pneumothorax, and genetic associations in 89 families with BirtHogg-Dube syndrome. Am J Respir Crit Care Med 2007;175:1044-53.

4. Pavlovich CP, Grubb RL 3rd, Hurley K, et al. Evaluation and management of renal tumors in the Birt-Hogg-Dube syndrome. J Urol 2005;173:1482-6.

5. Zbar B, Alvord WG, Glenn G, et al. Risk of renal and colonic neoplasms and spontaneous pneumothorax in the Birt-Hogg-Dube syndrome. Cancer Epidemiol Biomarkers Prev 2002;11:393-400. 